\title{
Impaired functional recovery of endothelial colony-forming cells from moyamoya disease in a chronic cerebral hypoperfusion rat model
}

\author{
*Seung Ah Choi, PhD, 1,2 Sangjoon Chong, MD, 1,2 Pil Ae Kwak, MS, ${ }^{1,2}$ Youn Joo Moon, MS,, \\ Anshika Jangra, MS, ${ }^{1,2}$ Ji Hoon Phi, MD, PhD, ${ }^{1,2}$ Ji Yeoun Lee, MD, PhD, ${ }^{1-3}$ \\ Sung-Hye Park, MD, PhD, ${ }^{4}$ and Seung-Ki Kim, MD, PhD ${ }^{1,2}$ \\ 'Division of Pediatric Neurosurgery, Pediatric Clinical Neuroscience Center, Seoul National University Children's Hospital; \\ 2Department of Neurosurgery, Seoul National University Hospital, Seoul National University College of Medicine; ${ }^{3}$ Department \\ of Anatomy, Seoul National University College of Medicine; and ${ }^{2}$ Department of Pathology, Seoul National University Hospital, \\ Seoul, Korea
}

OBJECTIVE Endothelial colony-forming cells (ECFCs) isolated from pediatric patients with moyamoya disease (MMD) have demonstrated decreased numbers and defective functioning in in vitro experiments. However, the function of ECFCs has not been evaluated using in vivo animal models. In this study, the authors compared normal and MMD ECFCs using a chronic cerebral hypoperfusion $(\mathrm{CCH})$ rat model.

METHODS A CCH rat model was made via ligation of the bilateral common carotid arteries (2-vessel occlusion [2-VO]). The rats were divided into three experimental groups: vehicle-treated $(n=8)$, normal ECFC-treated $(n=8)$, and MMD ECFC-treated $(n=8)$. ECFCs were injected into the cisterna magna. A laser Doppler flowmeter was used to evaluate cerebral blood flow, and a radial arm maze test was used to examine cognitive function. Neuropathological examinations of the hippocampus and agranular cortex were performed using hematoxylin and eosin and Luxol fast blue staining in addition to immunofluorescence with CD31, von Willebrand factor, NeuN, myelin basic protein, glial fibrillary acidic protein, and cleaved caspase-3 antibodies.

RESULTS The normal ECFC-treated group exhibited improvement in the restoration of cerebral perfusion and in behavior compared with the vehicle-treated and MMD ECFC-treated groups at the 12-week follow-up after the 2-VO surgery. The normal ECFC-treated group showed a greater amount of neovasculogenesis and neurogenesis, with less apoptosis, than the other groups.

CONCLUSIONS These results support the impaired functional recovery of MMD ECFCs compared with normal ECFCs in a $\mathrm{CCH}$ rat model. This in vivo study suggests the functional role of ECFCs in the pathogenesis of MMD.

https://thejns.org/doi/abs/10.3171/2018.8.PEDS1883

KEYWORDS moyamoya disease; endothelial colony-forming cells; chronic cerebral hypoperfusion; rat model; vascular disorders

$\mathrm{M}$ oyamoya disease (MMD) is a chronic cerebrovascular disorder characterized by a progressive occlusion of the major bilateral intracranial arteries. ${ }^{10}$ MMD has been identified as one of the important causes of stroke in children..$^{20}$ This chronic cerebrovascular disorder affects cognition, intelligence, memory, executive functioning, and quality of life. ${ }^{14}$ The associated cognitive impairment is generally linked to pathogenesis in the brain. MMD pathogeneses, such as inflammation, immune complex, upregulation of angiogenic factors, and abnormalities in endothelial colony-forming cells (ECFCs; previously termed endothelial progenitor cells), have been studied. ${ }^{13,17}$

ECFCs comprise a primitive cell type in the endothe-

ABBREVIATIONS 2-VO = 2-vessel occlusion; $\mathrm{CBF}=$ cerebral blood flow; $\mathrm{CCH}=$ chronic cerebral hypoperfusion; $\mathrm{ECFC}=$ endothelial colony-forming cell; $\mathrm{GFAP}=$ glial fibrillary acidic protein; $L D F=$ laser Doppler flowmeter; $L D L=$ low-density lipoprotein; $L F B=$ Luxol fast blue; $M B P=$ myelin basic protein; $M C A=$ middle cerebral artery; $M C A O=$ MCA occlusion; MMD = moyamoya disease; $R A M=$ radial arm maze; $S M P C=$ smooth muscle progenitor cell; $v$ vf $=$ von Willebrand factor . SUBMITTED February 6, 2018. ACCEPTED August 6, 2018.

INCLUDE WHEN CITING Published online November 9, 2018; DOI: 10.3171/2018.8.PEDS1883.

* S.A.C. and S.C. contributed equally to this work. 
TABLE 1. Information of normal volunteer and MMD patients

\begin{tabular}{|c|c|c|c|c|c|c|}
\hline Case No. & Age (yrs), Sex & Familial History & Involvement & Clinical Presentation & Suzuki Grade & Associated Conditions \\
\hline \multicolumn{7}{|c|}{ Normal control } \\
\hline 1 & $20, M$ & NA & NA & NA & NA & NA \\
\hline 2 & $25, \mathrm{M}$ & NA & NA & NA & NA & NA \\
\hline 3 & $24, F$ & NA & NA & NA & NA & NA \\
\hline 4 & $21, F$ & NA & NA & NA & NA & NA \\
\hline \multicolumn{7}{|l|}{ MMD } \\
\hline 1 & $11, \mathrm{M}$ & No & Bilat & Hemorrhage & Rt II, It III & None \\
\hline 2 & $17, \mathrm{~F}$ & No & Bilat & TIA & Rt III, It III & None \\
\hline 3 & $4, \mathrm{~F}$ & No & Bilat & $\mathrm{HA}, \mathrm{TIA}$ & Rt III, It III & Renovascular hypertension \\
\hline 4 & $6, M$ & No & Unilat & $\mathrm{TIA}$ & Rt I, It 0 & None \\
\hline
\end{tabular}

$\mathrm{HA}=$ headache; $\mathrm{NA}=$ not available; $\mathrm{TIA}=$ transient ischemic attack.

lial lineage that move to the site of an injury. ECFCs have been known to promote neovascularization, thus improving blood flow and tissue recovery. ${ }^{22}$ Circulating ECFCs in peripheral blood have robust angiogenic properties that are associated with MMD pathogenesis. ${ }^{19,21,30}$ The results of our previous study suggested that defective functioning of ECFCs is related to MMD. ${ }^{21}$ However, the function of MMD ECFCs has not been fully clarified because animal models are limited in their reproduction of the complex process of MMD.

Chronic cerebral hypoperfusion $(\mathrm{CCH})$ is a cerebrovascular disease that exhibits a correlation between the severity of memory dysfunction and a decline in cerebral blood flow (CBF). ${ }^{1,3}$ Animal models of permanent bilateral occlusion of both common carotid arteries (2-vessel occlusion [2-VO]) have been extensively utilized to study the consequences of neurocognitive dysfunction with reduced CBF.? Studies have reported the role of normal ECFCs and have suggested the therapeutic potential of these cells in animal models of vascular disease, ${ }^{6,22}$ including a $\mathrm{CCH}$ rat model.$^{11}$ However, there has been little to no research on the functional impairment of MMD ECFCs in animal disease models.

In the present study, we investigated the biological dysfunction of MMD ECFCs in a CCH rat model. Our results might provide further insight into the effect of ECFCs in MMD pediatric patients on neovasculogenesis and neurogenesis.

\section{Methods \\ ECFC Cultures}

Blood samples from healthy normal volunteers $(n=4)$ and MMD patients $(n=4)$ were obtained (Table 1$)$ with informed consent after Seoul National University Hospital IRB approval. All MMD patients had confirmed diagnoses based on cerebral angiography. Isolated cells were cultured at $37^{\circ} \mathrm{C}$ with $5 \% \mathrm{CO}_{2}$ in a humidified atmosphere. The cells were used under cell passage no. 6 .

\section{ECFC Characterization}

ECFCs were characterized by morphological confirmation, acetylated low-density lipoprotein (LDL) uptake, flow cytometry analysis using antibodies (KDR, CD34, CD31, CD45, and CD14), and tubule formation assays, as described in our previous report. ${ }^{21}$ Observation of ECFC morphology was performed using a bright-field microscope (Leica Microsystems).

For LDL uptake, ECFCs $\left(1 \times 10^{3}\right)$ were seeded in a chamber slide (Nunc, Lab-Tek) with the endothelial growth media for 24 hours. On the next day, the media was replaced with endothelial growth media containing $10 \mu \mathrm{g} / \mathrm{ml}$ 1,10-dioctadecyl-3,3,30,30-tetramethylindocarbocyaninelabeled LDL (Dil-Ac-LDL, Biomedical Technologies) and incubated for 4 hours at $37^{\circ} \mathrm{C}$ with $5 \% \mathrm{CO}_{2}$. ECFCs were washed with phosphate-buffered saline and fixed in 3\% formaldehyde for 15 minutes. LDL uptake was visualized by fluorescence microscopy (Leica Microsystems).

For flow cytometry analysis, ECFCs $\left(1 \times 10^{6}\right)$ were incubated for staining with phycoerythrin-conjugated anti-human KDR, CD34, CD31, CD45, and CD14 antibodies (BD Biosciences). The data were analyzed using a FACScan flow cytometer and CellQuest software (BD Biosciences).

For capillary tube formation assay in vitro, ECFCs (2 $\times 10^{4}$ ) were plated in a Matrigel-coated 48-well plate and incubated for 18 hours. The number of tubes was counted at 4 random microscopic fields by two independent observers.

\section{Experimental Design}

Figure 1 shows a schematic plot of the experimental design. Four-week-old rats were brought in and allowed to spend a week adapting; they were given radial arm maze (RAM) practice at 5 weeks old. The rats practiced for a total of 6 RAM tests for 2 weeks before surgery. The rats underwent 2-VO surgery at 7 weeks old (marked as "0" in the schematic diagram of Fig. 1) and ECFC treatment at 8 weeks old (marked as "1" in Fig. 1). The rats underwent laser Doppler flowmeter (LDF) monitoring before and after 2-VO surgery, which marked time point zero. The 2-VO procedure was confirmed via changes in $\mathrm{CBF}$ between baseline and after surgery. After surgery, the rats were given a few days to rest and recuperate, followed by the commencement of the first RAM test. At the begin- 


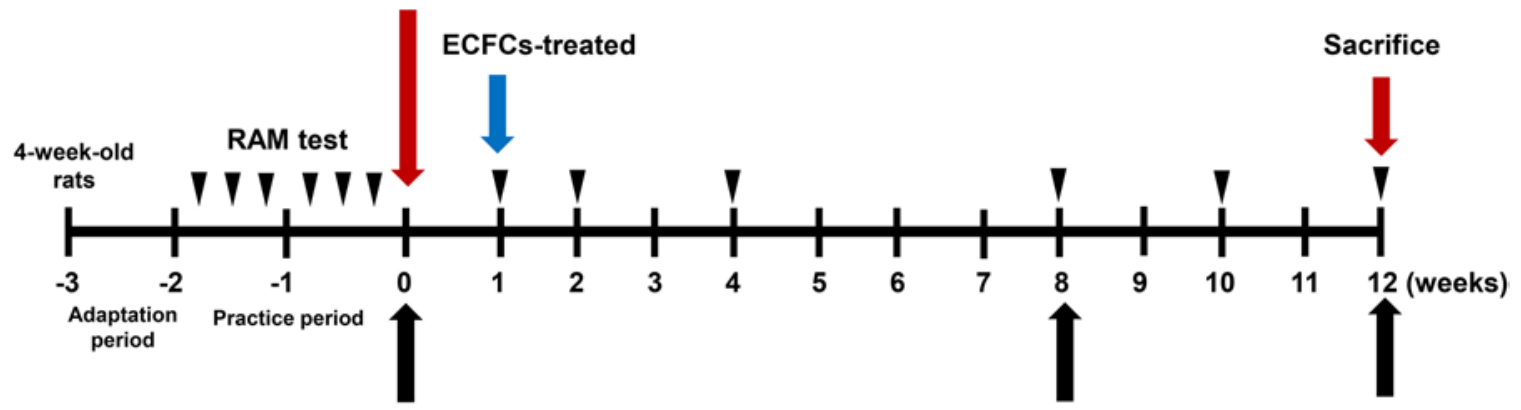

\section{LDF monitoring}

FIG. 1. Schematic diagram of the experimental design. Before the 2-VO surgery the rats underwent 6 sets of practice over 2 weeks to habituate to the maze test. After the 2-VO surgery CBF was estimated by LDF at 0,8 , and 12 weeks. Cognitive function was evaluated through a RAM test $1,2,4,8,10$, and 12 weeks after the 2-VO surgery. Normal and MMD ECFCs were injected into the cisterna magna the 1 st week after the 2-VO surgery.

ning of the first week postsurgery, vehicle-treated (saline), normal, or MMD ECFCs were administered to these rats. The RAM test was conducted 2, 4, 8, 10, and 12 weeks after the 2-VO surgery.

\section{Surgical Procedures of a CCH Rat Model Involving 2-VO}

All procedures involving animal experiments were approved by the Institutional Animal Care and Use Committee of Seoul National University Hospital Biomedical Research Institute. Wistar rats (5-week-old males, Orient Bio Inc.; approximately 2-3 rats per cage) were kept on a 12-hour light/dark cycle at a constant temperature of $22^{\circ} \mathrm{C}$ $\pm 1^{\circ} \mathrm{C}$ with free access to food and water except when performing the RAM test. For 2-VO surgery, the rats (7 weeks old) were fixed on the board in a supine position under anesthesia with a solution of $20 \mathrm{mg} / \mathrm{kg}$ zolazepam (Virbac) and $10 \mathrm{mg} / \mathrm{kg}$ xylazine (Bayer). After making an approximately $2-\mathrm{cm}$ incision in the skin, the bilateral common carotid arteries were exposed by isolating them from the vagus nerve and its sheath. ${ }^{7,23}$ The arteries were double-ligated with $4-0$ silk $8-10 \mathrm{~mm}$ below the visible region of the common carotid artery.

\section{Injection of ECFCs}

One day prior to injection of the ECFCs, the rats were administered an immunosuppressant $(8 \mathrm{mg} / \mathrm{kg}$ cyclosporin A dissolved in olive oil; Abcam) daily via intraperitoneal injection for 6 weeks. One week after the 2-VO surgery, the rats were randomly divided into three groups (24 rats total, 8 rats per group) as follows: a vehicle-injected (saline) group, a normal ECFC-treated group, and an MMD ECFC-treated group. The ECFCs were labeled with fluorescent spherical silica nanoparticles with CELLSTALKER-CSR (Biterials), according to the manufacturer's protocol. The rats were anesthetized via intraperitoneal administration of a mixture of $20 \mathrm{mg} / \mathrm{kg}$ zolazepam and $10 \mathrm{mg} / \mathrm{kg}$ xylazine. The skin above the posterior atlantooccipital membrane was cut, and the muscular layers were moved aside in a stereotactic frame (Harvard Apparatus). The tip of a Hamilton syringe (30-gauge) was connected to an intrathecal catheter (ALZET Osmotic Pumps) and filled with the ECFCs. The tip of the intrathecal catheter was carefully inserted into the cisterna magna through the posterior atlantooccipital membrane. The ECFCs $\left(6 \times 10^{5}\right.$ cells in $30 \mu \mathrm{l}$ of saline) were injected at a rate of $1 \mu \mathrm{l} / \mathrm{min}$ using an infusion pump (Harvard Apparatus). The muscular layers were realigned, and the skin was sutured.

\section{Laser Doppler Flowmeter}

CBF was measured using an LDF (PeriFlux System 5000 , Perimed AB) with the relative flow value expressed in perfusion units before and after 2-VO surgery, which used a flexible probe placed over the skull, as described previously. ${ }^{8,32}$ The rats ( 8 per group) were anesthetized using $4 \%$ isoflurane and were maintained under $1 \%-3 \%$ oxygen. The tip of the LDF probe (1.0-mm diameter, probe 407; a small, straight probe) was placed perpendicular to the cerebral cortex with a probe holder. It was attached to the cranial window of the barrel cortex in an area that was free of larger blood vessels in order to minimize any interference with the signal. The LDF flux signal that was proportional to the tissue perfusion/CBF at the measurement point was calculated and recorded with the Perisoft software (Perimed AB), and spectrographic data were collected for each group. The LDF flux signal was stabilized and measured 30 seconds before and after the occlusion surgery. The magnitude of the evoked CBF was calculated as the mean percentage change relative to baseline. Measurements were performed at four different time points per subject: preoperatively, immediately postoperatively, and at 8 weeks and 12 weeks after 2-VO surgery.

\section{Eight-Arm Radial Maze}

The memory impairment of the rats (8 per group) was assessed via an 8 -arm RAM, as previously described. ${ }^{27,28}$ The rats were food deprived to $85 \%$ of their free-feeding weight for the RAM. Briefly, the rats were placed in the center of the maze, and the test was conducted for $5 \mathrm{~min}$ utes to determine if the rats ate all the feed. Before the 2-VO surgery, the rats were trained 6 times over 2 weeks. Once released, the rats were free to forage in the maze until they consumed all the accessible food or until 3 min- 
utes had elapsed. The sequence of the arms entered and the latency to find all four food rewards were recorded and calculated for every trial. The time taken to eat all the food and the number of trial errors were recorded. Trial errors were considered to have occurred if the rat went to an arm without feeding on the pellet or returned to an arm already visited during the trial. Only the median trial was selected for analysis to control for any learning effect on the subsequent trials.

\section{Histological and Immunofluorescence Analyses}

The rats (8 per group) were perfused and killed with $4 \%$ formaldehyde under deep anesthesia 12 weeks after the 2-VO surgery. Whole-brain tissues were prepared as frozen sections for histological and immunofluorescence analyses, as previously described. ${ }^{4}$

For the histological analysis, sections were stained with either $\mathrm{H} \& \mathrm{E}$ or Luxol fast blue (LFB) for myelin staining, as described previously. ${ }^{33}$ At the same exposure level, 3 sections (per rat) were obtained, and 3 sections that were randomly chosen were observed using light microscopy. Quantitative analysis of histological changes was performed by two blinded observers and inspected by a pathologist professor (S.H.P.).

For distribution analysis, CELL-STALKER-CSR-labeled normal ECFCs were evaluated 4 weeks after ECFC injection in injured rats $(n=3)$ using fluorescence microscopy.

For the immunofluorescence analysis, sections were incubated with anti-CD31 (1:100, Abcam), anti-von Willebrand factor (vWF; 1:100, Abcam), anti-NeuN (1:50, Millipore), anti-myelin basic protein (MBP; 1:500, Abcam), anti-glial fibrillary acidic protein (GFAP; 1:500, Abcam), and anti-cleaved caspase-3 (1:100, Millipore). The Alexa-488 (1:400, Invitrogen) and Alexa-594 (1:400, Invitrogen) secondary antibodies were used. Positive cells that had identifiable nuclei and that were counterstained with DAPI within the hippocampal CA1 area in 3 randomly chosen sections per rat were averaged for quantification. The mean value of the 5 hot-spot counts was used in the following statistical analyses. Quantitative analysis of immunofluorescence was performed by two observers blinded to all groups through data collection.

\section{Statistical Analysis}

Statistical analyses were conducted with GraphPad Prism software. All experimental data are given as the mean and standard deviation. Repeated-measures ANOVA with a post hoc correction for multiple comparisons (Bonferroni) was used for analysis among the three groups. Significant differences were considered at $\mathrm{p}<$ 0.05 . All in vitro experiments were conducted in triplicate.

\section{Results}

\section{Identification of ECFCs Derived From Normal Volunteers and MMD Patients}

First, we characterized the ECFCs obtained from the normal volunteers and MMD patients. Typical endothelial cobblestone morphology of the late ECFCs (Fig. 2A) and positive LDL uptake (Fig. 2B and D) were observed in both the normal and MMD ECFCs. However, we found that the normal ECFCs formed capillary tubes better than the MMD ECFCs in vitro (Fig. 2C and E, $\mathrm{p}<0.001$ ).

A flow cytometry analysis showed that viable cells from the ECFCs were uniformly positive for KDR, CD34, and CD31 but were negative for CD45 and CD14 (Fig. 2F, Supplemental Table S1). There were no differences in morphology, LDL uptake, or surface marker expression between the normal and MMD ECFCs in vitro.

\section{Establishment of the CCH Rat Model and Distribution of Normal ECFCs}

The rat survival rate was $63 \%(24 / 38)$ within the 72 hours following the 2-VO surgery. All 14 deaths occurred within the first 72 hours after the ischemic insult. The rats that survived exhibited decreased body weight or transient difficulties in feeding. We tested a total of 24 rats (8 rats per group) at the outset and analyzed these rats.

We observed the distribution of normal ECFCs after injection of the ECFCs labeled with CELL-STALKERCSR into the cisterna magna. The fluorescence results demonstrated that positive cells were found in the hippocampal CA1 area and agranular cortex 4 weeks after the 2-VO surgery (Fig. 3).

\section{Effect of ECFCs on Cerebral Perfusion}

The CBF dropped rapidly to $54.5 \% \pm 22.9 \%$ of the baseline level in all rats 1 hour after the 2-VO surgery. We found that the CBF values gradually recovered over 8 weeks in all groups. Interestingly, the CBF value was significantly higher in the normal ECFC-treated group $(110.1 \% \pm 13.4 \%)$ than in the vehicle-treated $(61.8 \% \pm$ $25.6 \%, \mathrm{p}<0.001)$ and MMD ECFC-treated $(55.5 \% \pm$ $17.3 \%, \mathrm{p}<0.001$ ) groups at 12 weeks (Fig. 4). The CBF values did not significantly differ between the vehicletreated and MMD ECFC-treated groups at any time point.

\section{Effect of ECFCs on Cognitive Function}

Excluding the habituation period, the assessments of learning performance began 1 week after the 2-VO surgery. The RAM results revealed that the $2-\mathrm{VO}$ rats injected with normal ECFCs exhibited significantly shorter latency times $(263.6 \pm 21.9$ seconds [vehicle] vs $155.0 \pm 51.6$ seconds [normal ECFC] vs $272.8 \pm 37.5$ seconds [MMD ECFC], $\mathrm{p}<0.001)$ and fewer trial errors $(22.6 \pm 4.3$ times [vehicle] vs $10.1 \pm 6.2$ times [normal ECFC] vs $25.0 \pm 2.7$ times [MMD ECFC], $\mathrm{p}<0.001$ ) than the vehicle-treated or MMD ECFC-treated groups at 12 weeks (Fig. 5). This result showed that the normal ECFC-treated group recovered from the learning impairment. The changes in the RAM results between the vehicle-treated and MMD ECFC-treated groups were not significantly different in terms of the latency times $(p>0.05)$ and trial errors $(p>0.05)$.

\section{Effects of ECFCs on Neurons and Myelin in the Hippocampal CA1 Area and the Agranular Cortex}

The hippocampal CA1 area and the prefrontal agranular cortex are closely associated with cognitive function. First, we performed $\mathrm{H} \& \mathrm{E}$ staining to evaluate neuronal loss in the rats. There was extensive damage in the ve- 
Choi et al.

A
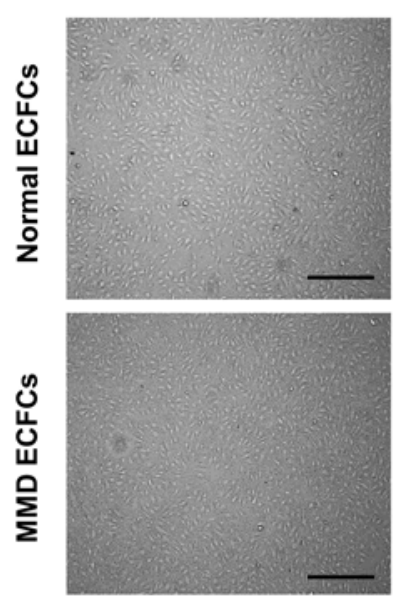

B
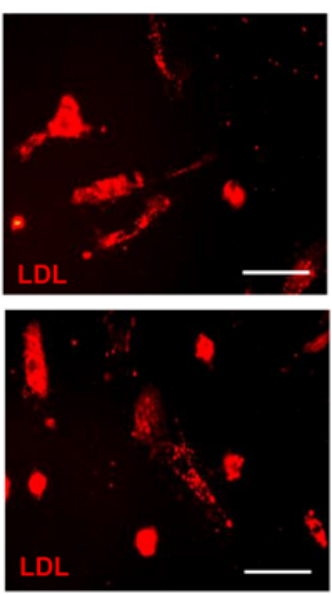

C
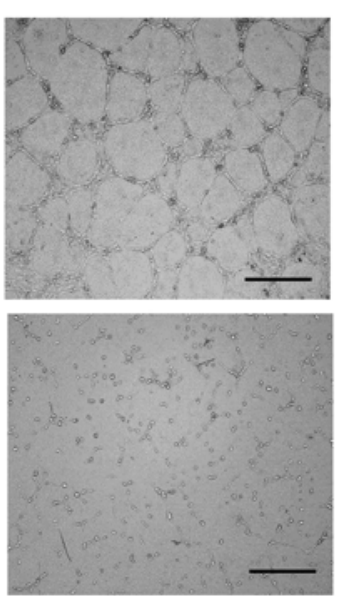

D

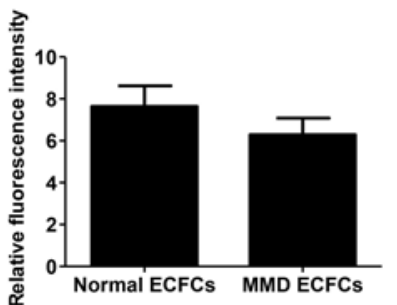

E

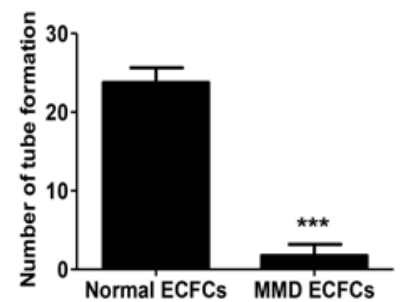

$\mathbf{F}$

KDR
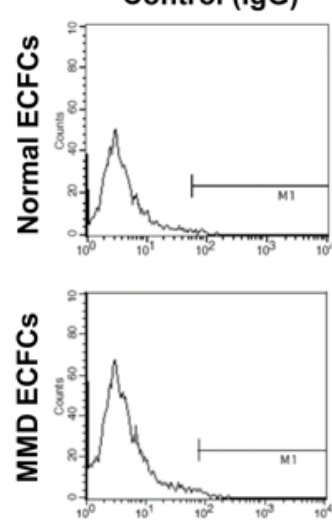
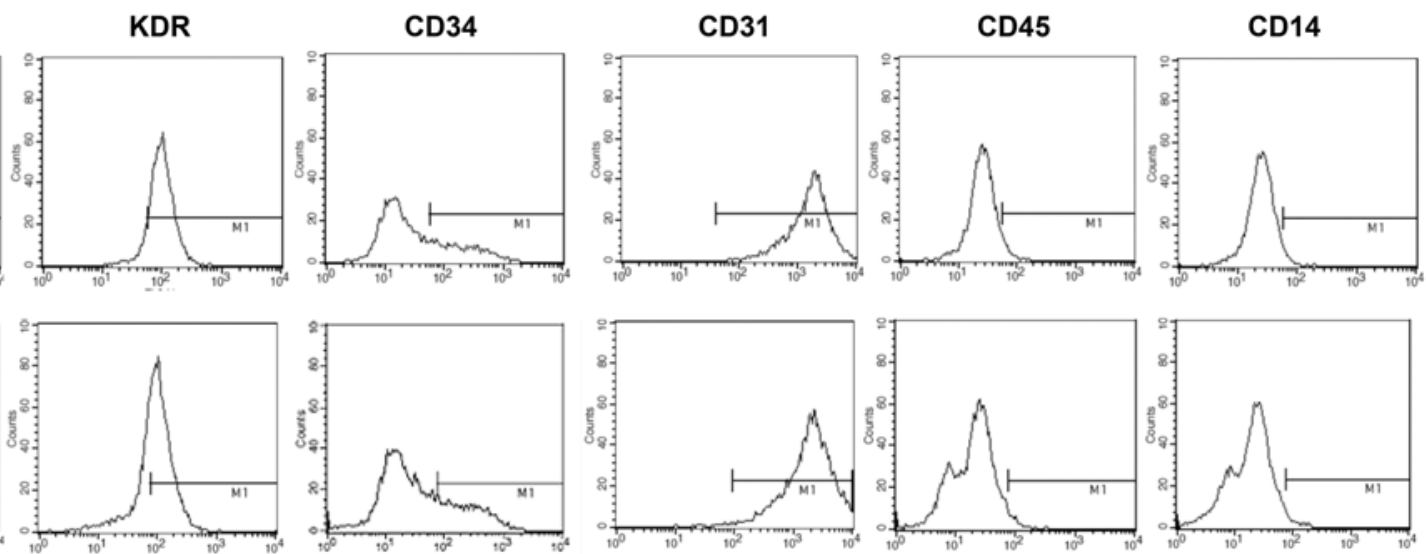

FIG. 2. Characterization of ECFCs from normal volunteers and MMD patients. A: Peripheral blood mononuclear cells from both normal and MMD ECFCs differentiated into a cobblestone-like morphology. Bar $=500 \mu \mathrm{m} . \mathrm{B}$ and $\mathrm{D}$ : The uptake of acetylated LDL of both normal and MMD ECFCs. Bar $=100 \mu \mathrm{m}$. C and E: Capillary network formation on Matrigel showing that normal ECFCs have better capillary formation abilities than the MMD ECFCs. ${ }^{* \star *} p<0.001$. Bar $=500 \mu \mathrm{m}$. F: Representative flow cytometry analysis of ECFCs revealing high expression of the surface markers KDR, CD34, and CD31, but low expression of CD45 and CD14.
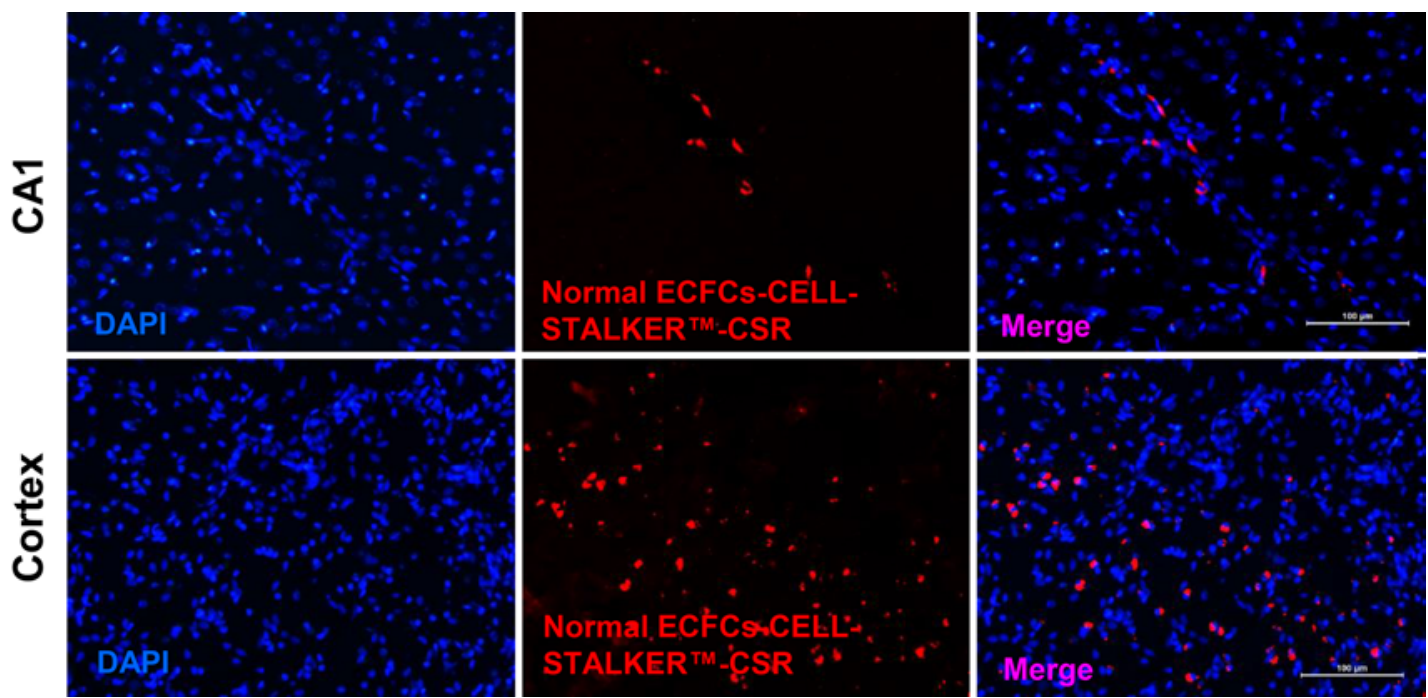

FIG. 3. Distribution of normal ECFCs in the hippocampal CA1 area and agranular cortex. Representative fluorescence images showing that CELL-STALKER-CSR-labeled normal ECFCs (red) were observed 4 weeks after injection of the cells. Nuclei were counterstained with DAPI (blue). Bars $=100 \mu \mathrm{m}$. 


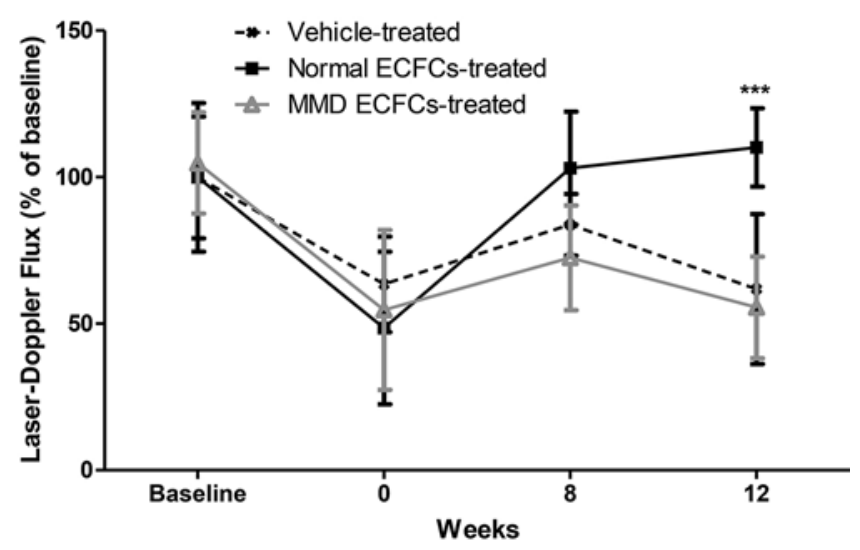

FIG. 4. Measurement of CBF by LDF at the indicated time intervals. CBF was rapidly reduced in all rats to $54.5 \% \pm 22.9 \%$ of the baseline level immediately after the 2-VO surgery and gradually recovered over 8 weeks in all rats. CBF was significantly higher in the normal ECFC-treated group than in the vehicle-treated group $(61.8 \% \pm 25.6 \%)$ and MMD ECFC-treated group $(55.5 \% \pm 17.3 \%)$ at 12 weeks. Values are presented as mean \pm SD. ${ }^{* * *} p<0.001$.

hicle-treated group and MMD ECFC-treated group, with disfigured and distorted shapes of the nuclei in comparison with those of the normal ECFC-treated group, which had uniformly shaped nuclei and cytoplasm in the CA1 area and the agranular cortex (Fig. 6A). The vehicle-treated group and MMD ECFC-treated group were shown to exhibit vacuoles and the disappearance of both myelinated fibers and nerve cells via LFB staining, but the normal ECFC-treated group demonstrated significantly less neuronal damage (Fig. 6B).

\section{Effect of ECFCs on Neovasculogenesis, Neurogenesis, and Apoptosis in the Hippocampal CA1 Area}

After treatment with injection of the ECFCs into the cisterna magna of the $\mathrm{CCH}$ rats, the biological factors related to neovasculogenesis, neurogenesis, and apoptosis were evaluated via immunofluorescence analyses (Fig. 7, Supplemental Table S2). First, we observed the neovasculogenesis of the ECFCs. The normal ECFC-treated group showed a significantly greater amount of CD31and vWF-positive cells than the vehicle-treated group ( $p$ $<0.01)$ and MMD ECFC-treated group ( $<$ 0.05). Next, neovasculogenesis was more prominent in the normal ECFC-treated group. The normal ECFC-treated group demonstrated more NeuN- and MBP-positive cells than the vehicle-treated group $(\mathrm{p}<0.001)$ and MMD ECFCtreated group $(\mathrm{p}<0.05)$. In contrast, the GFAP expression levels were similar across groups. Finally, we found that the MMD ECFC-treated group exhibited a greater number of cleaved caspase-3-positive cells marked for apoptosis than the control vehicle-treated group $(\mathrm{p}<0.05)$ and normal ECFC-treated group $(\mathrm{p}<0.01)$.

\section{Discussion}

In this study, we evaluated the therapeutic effect of ECFCs of normal volunteers and MMD patients on chronic hypoperfusion in a $\mathrm{CCH}$ rat model. Restoration

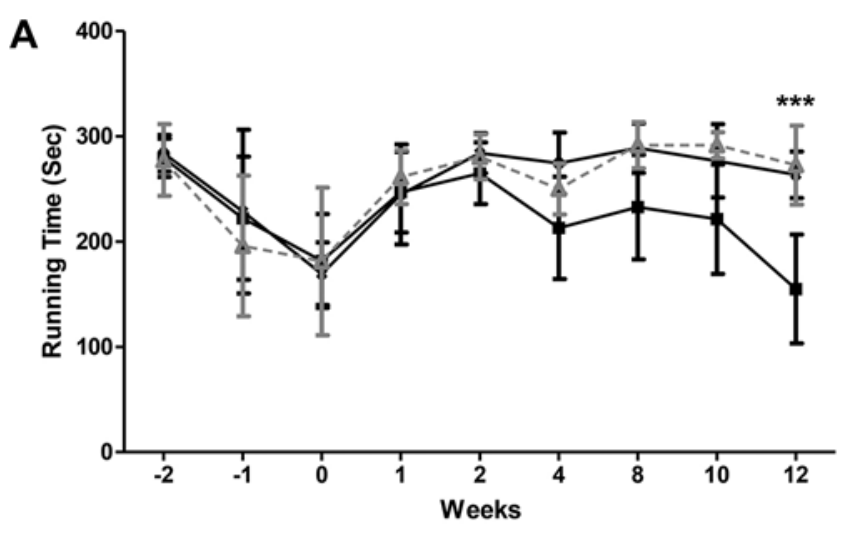

- - Vehicle-treated $\rightarrow$ - Normal ECFCs-treated $-\Delta$ MMD ECFCs-treated

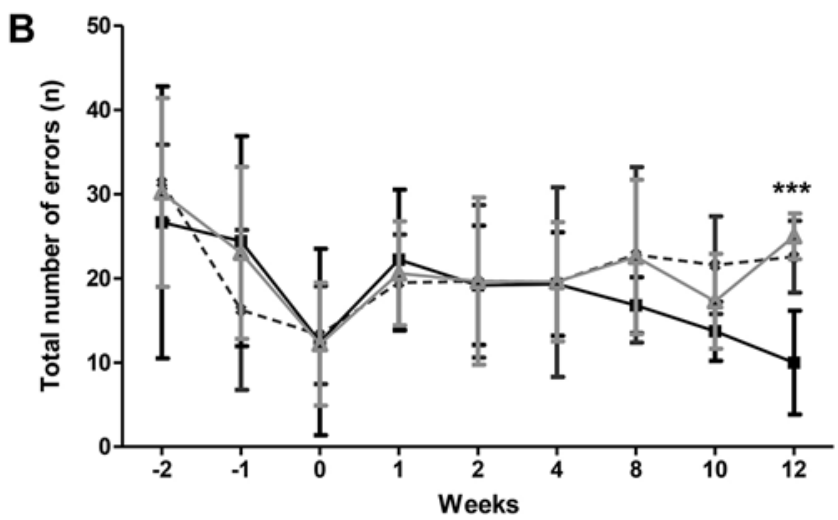

FIG. 5. Effect of ECFCs on cognitive function. A: The running time after 2-VO surgery was found to be significantly lower in the normal ECFCtreated group than in the vehicle-treated group and MMD ECFC-treated group. ${ }^{* *} p<0.001$. B: The total number of errors was also significantly lower in the normal ECFC-treated group than in the vehicle-treated group and MMD ECFC-treated group. Values are presented as mean \pm SD. ${ }^{* * *} p<0.001$.

of cerebral perfusion and improved learning ability were observed in the normal ECFC-treated group but not in the control vehicle-treated group or MMD ECFC-treated group at 12 weeks. These results were confirmed by pathological examination, namely, an increase in CD31- and NeuN-positive cells and a decrease in cleaved caspase-3positive cells.

Despite the extensive efforts that have been made, an experimental animal model of MMD has not yet been established. Various animal models using inflammatory and immunological reactions, such as a serum sickness model, a Propionibacterium acnes-infected model, and an $\mathrm{N}$ acetylmuramyl-L-alanyl-D-isoglutamine injection model, have been designed, but none of them exhibit any stenotic lesions in the cerebral artery or the development of collateral vessels. ${ }^{5,17,18}$ After the recent identification of ring finger protein 213 (RNF 213) as a susceptible gene for MMD through genetic studies ${ }^{16}$ RNF 213 -deficient homozygous knockout mice were studied. Unfortunately, no stenoocclusive lesions of the cerebral vasculature developed in this model, as they did in the other models. ${ }^{31}$

As it is impossible to reproduce MMD itself in animals, the vascular occlusion model is a good alternative. 
A

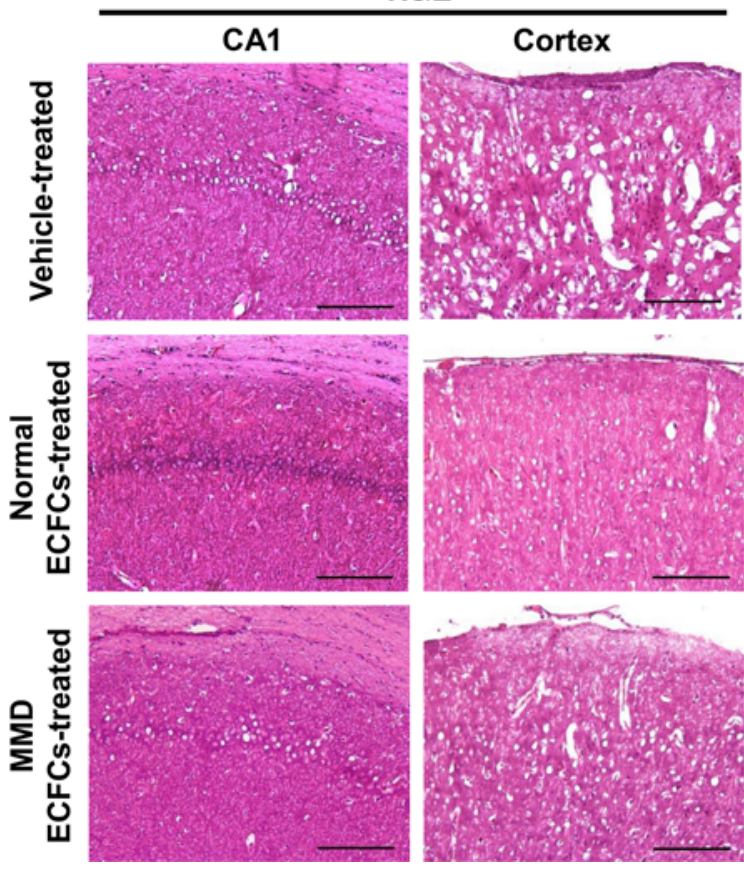

B
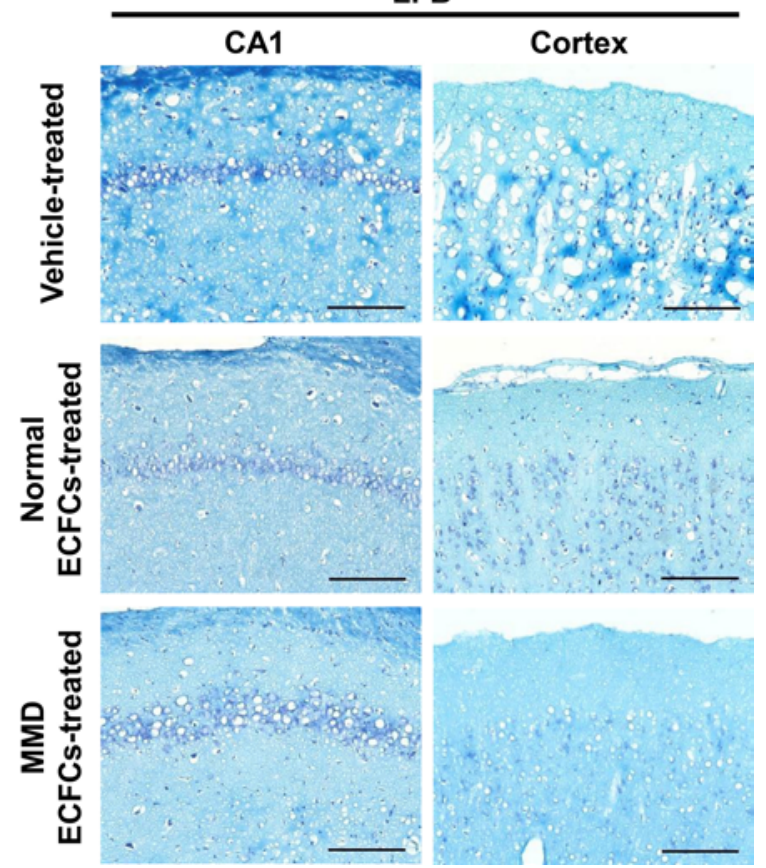

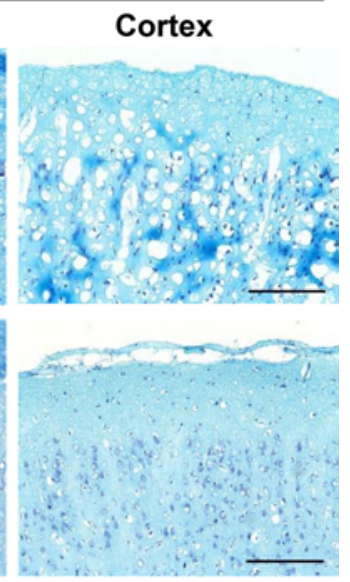

LFB

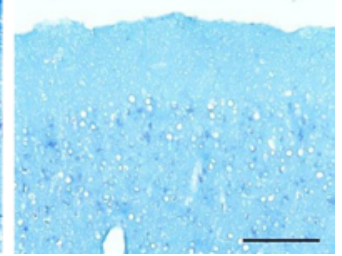

FIG. 6. Effects of ECFCs on neurons and myelin in the hippocampal CA1 area and agranular cortex. H \& E (A) and LFB (B) staining were performed on sections from the hippocampal CA1 region and the agranular cortex after injection of ECFCs. Compared with the normal ECFC-treated group, $\mathrm{H}$ \& E staining showed a loss of hippocampal CA1 pyramidal neurons in the MMD ECFCtreated group. LFB staining revealed reduced demyelination in the normal ECFC-treated group. Blue areas indicate myelination, whereas pale areas indicate demyelination. Bar $=100 \mu \mathrm{m}$.

The middle cerebral artery (MCA) occlusion (MCAO) model and the 2-VO model are representative animal models that use occlusion in rodents. The MCAO model is widely used for the study of acute stroke and reperfusion injuries because it forms a lesion immediately after occlusion without collateral damage. ${ }^{9}$ On the other hand, with the 2-VO model, a chronic hypoperfusion state arises from decreased CBF. This creates a similar hemodynamic environment to that present in MMD. The 2-VO model has been used primarily to study chronic diseases such as neurodegenerative disease and vascular dementia, and it has also been used as a model of MMD., ${ }^{3,734}$ Although the 2-VO model is not progressive, a persistent decrease, but not a complete cessation, in perfusion around the circle of Willis in this model is analogous to the hemodynamic status observed in MMD. In this study, we selected a CCH animal model with 2-VO in rats for the study of MMD. Although it is not identical and has some limitations, we believe that, currently, the 2-VO model is a suitable one for inferring the function of ECFCs in MMD, and it is henceforth useful. Wistar rats are ideal for use in a chronic hypoperfusion model via 2-VO because they have a significantly greater number of proximal (small-caliber) side branches in the long proximal MCA segment compared with other rats. ${ }^{2,734}$

Because of the lack of an in vivo model, previous studies of ECFCs in MMD were performed in vitro. A Matrigel plug assay was the only type of in vivo study of MMD ECFCs that had been conducted until now. ${ }^{21}$ Although a
Matrigel plug assay can be performed in vivo, it is a simple method that solely focuses on identifying neovasculogenesis in the localized environment under the influence of a foreign matrix. Furthermore, the subjects used for Matrigel plug assays were not designed to mimic MMD. The previous MMD studies using the $\mathrm{CCH}$ model in rats evaluated the effect of gene therapy under the ischemic status induced by the model, but they did not evaluate MMD ECFC function itself. ${ }^{12}$ In this study, it is meaningful that the function of MMD ECFCs was evaluated in comparison with the in vivo animal model.

Here, we investigated the impaired functional recovery of MMD ECFCs in vivo. Our data demonstrated that normal ECFCs have a protective effect on cognitive impairment induced by chronically decreased cerebral perfusion with a delayed recovery of blood flow, whereas the MMD ECFCs exhibited no effect without a recovery in the $\mathrm{CCH}$ rat model. Upon histopathological examination-including immunofluorescence analysis of the hippocampus and agranular cortex of the subjects-induced neovasculogenesis, preserved neuronal cells, and decreased apoptosis were evident in the normal ECFC-treated group. In contrast, impaired neovasculogenesis, neuronal loss, and apoptosis were observed in the MMD ECFC-treated group, similar to the vehicle-treated group. The neuroprotective effect of the ECFCs caused by neovasculogenesis has been consistently addressed and has been studied as a treatment for ischemic stroke..$^{22,24}$ The results of our study showed that MMD ECFCs lack this capability. 
A

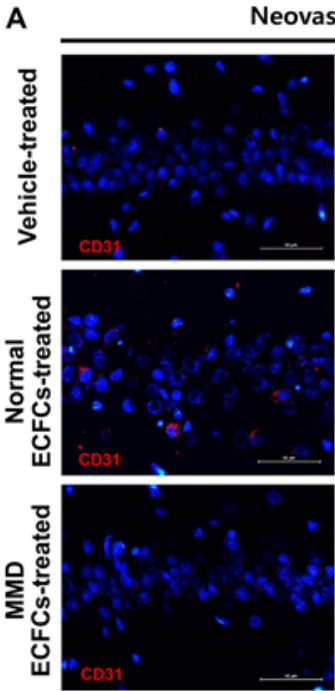

B

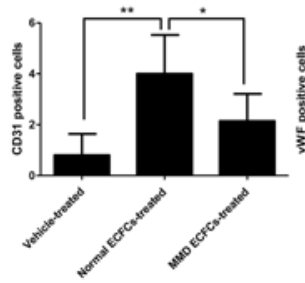

eovascularization
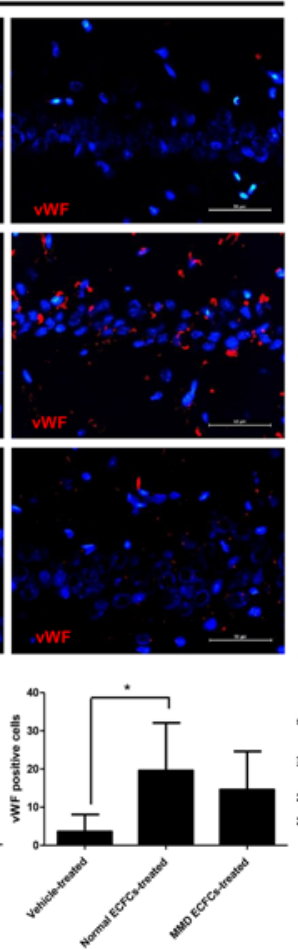
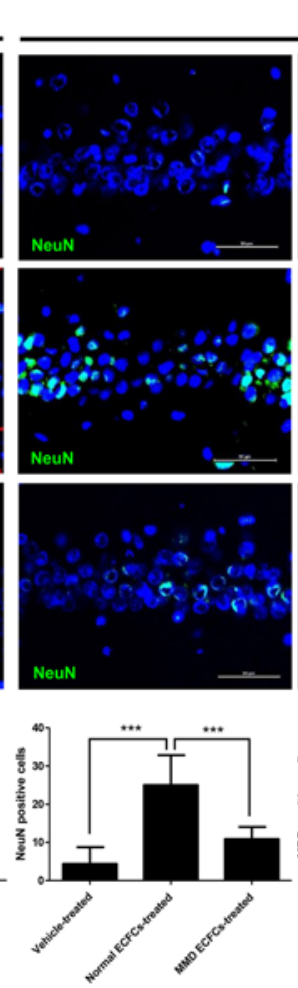

Neurogenesis
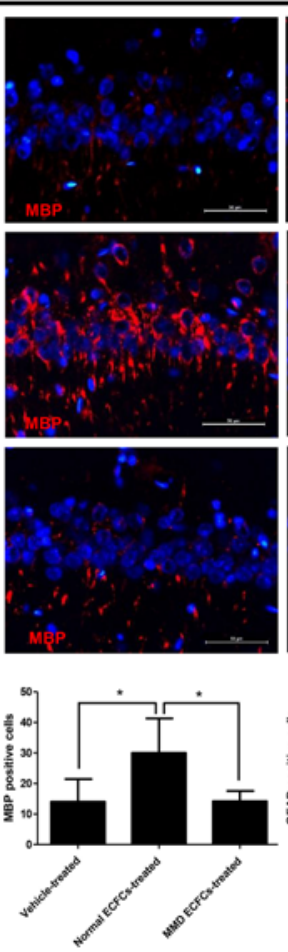
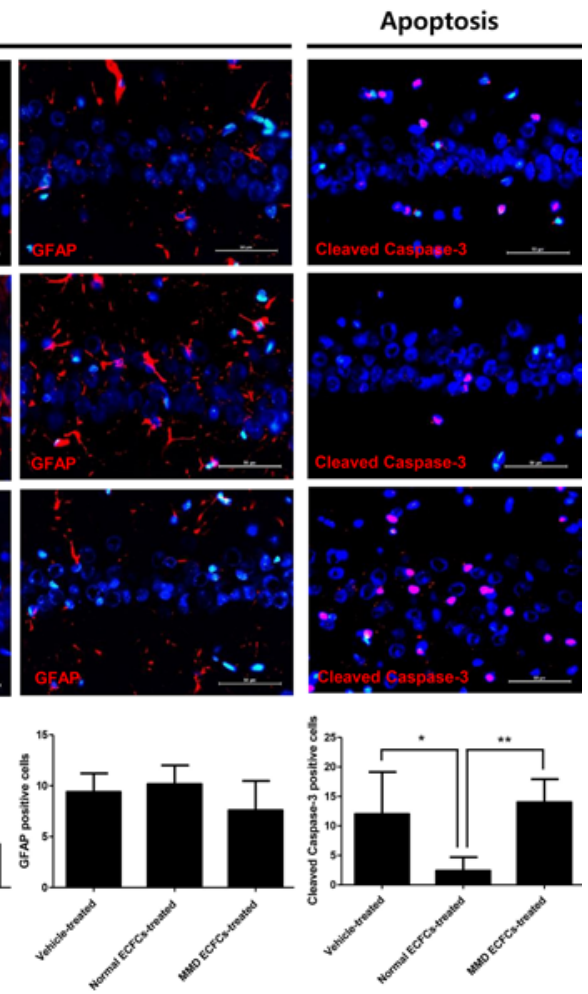

FIG. 7. Effects of ECFCs on neovasculogenesis, neurogenesis, and apoptosis in the hippocampal CA1 region. A: Representative immunofluorescence images show the expression of CD31, vWF, NeuN, MBP, GFAP, and cleaved caspase-3. The normal ECFC-treated group was compared with the vehicle-treated group and MMD ECFC-treated group. B: Quantification of the number of positive cells is shown in the graphs. The number of CD31- (red), vWF- (red), NeuN- (green), and MBP-positive (red) cells was significantly higher in the normal ECFC-treated group. The GFAP expression (red) shows that there were no statistically significant differences among the groups. A decreased number of cleaved caspase-3-positive (red) cells was observed in the normal ECFCtreated group. Bar $=50 \mu \mathrm{m}$. Nuclei were counterstained with DAPI (blue). ${ }^{*} p<0.05,{ }^{* *} p<0.01,{ }^{* * *} p<0.001$.

Increasing evidence has suggested that ECFCs play an important role in the pathological neovascularization in patients with MMD and that this role involves the dynamic interplay between arterial occlusion and neovascularization. ${ }^{15,19}$ Because of the complex pathophysiology of this disorder and limited techniques,,$^{29}$ there is still some controversy regarding the characterization of ECFCs in MMD. ${ }^{15,19,26}$ A previous study showed a reduced number of colony-forming units in ECFCs and a higher yield of outgrowth cells in MMD. ${ }^{15}$ These characteristics can reflect the complicated conditions of vascular occlusion and abnormal neovascularization in MMD. In our study, we reconfirmed a decrease both in the number of colonyforming units and in the tubule formation ability in MMD ECFCs compared with normal ECFCs.

In addition to the ECFC abnormalities, smooth muscle progenitor cells (SMPCs) and chemokines between the two cells are also thought to play a role in intimal thickening and proliferation of smooth muscle, which are characteristic of MMD. ${ }^{19,25}$ Experiments on MMD SMPCs or other chemokines were not performed in this study, and these aspects need to be addressed in future studies. However, because it is believed that MMD ECFCs play a major role in the dysfunction of both ECFCs and SMPCs and the interaction between them, rather than MMD SMPCs, ${ }^{25}$ it is important to provide a basis for future studies by identifying the functional abnormalities of MMD ECFCs in vivo using animal models. In our study we applied the 2-VO rat model, which is a suitable one for inferring the function of ECFCs in MMD, $3,7,34$ and we first identified and verified the abnormal and decreased angiogenic potential of MMD ECFCs in this rat model.

\section{Conclusions}

In this study we suggested the defective function of MMD ECFCs in a CCH rat model. Our results implied that abnormalities of ECFCs in terms of neovascularization and neurogenesis might lead to MMD. The fine-tuning of an animal model that can reproduce the dysfunction in MMD would be meaningful as it could be a cornerstone to the study of the pathophysiology and therapeutic targets of this disease.

\section{Acknowledgments}

This research was supported by a grant from the Korea Health Technology R\&D Project through the Korea Health Industry Development Institute, funded by the Ministry of Health \& Welfare, Republic of Korea (grant no. HI12C0066). 


\section{References}

1. Bennett SA, Tenniswood M, Chen JH, Davidson CM, Keyes MT, Fortin T, et al: Chronic cerebral hypoperfusion elicits neuronal apoptosis and behavioral impairment. Neuroreport 9:161-166, 1998

2. Berardi GR, Rebelatto CK, Tavares HF, Ingberman M, Shigunov P, Barchiki F, et al: Transplantation of SNAP-treated adipose tissue-derived stem cells improves cardiac function and induces neovascularization after myocardium infarct in rats. Exp Mol Pathol 90:149-156, 2011

3. Cechetti F, Pagnussat AS, Worm PV, Elsner VR, Ben J, da Costa MS, et al: Chronic brain hypoperfusion causes early glial activation and neuronal death, and subsequent long-term memory impairment. Brain Res Bull 87:109-116, 2012

4. Choi SA, Yun JW, Joo KM, Lee JY, Kwak PA, Lee YE, et al: Preclinical biosafety evaluation of genetically modified human adipose tissue-derived mesenchymal stem cells for clinical applications to brainstem glioma. Stem Cells Dev 25:897-908, 2016

5. Ezura M, Fujiwara S, Nose M, Yoshimoto T, Kyogoku M: Attempts to induce immune-mediated cerebral arterial injury for an experimental model of moyamoya disease. Childs Nerv Syst 8:263-267, 1992

6. Fan Y, Shen F, Frenzel T, Zhu W, Ye J, Liu J, et al: Endothelial progenitor cell transplantation improves long-term stroke outcome in mice. Ann Neurol 67:488-497, 2010

7. Farkas E, Luiten PG, Bari F: Permanent, bilateral common carotid artery occlusion in the rat: a model for chronic cerebral hypoperfusion-related neurodegenerative diseases. Brain Res Brain Res Rev 54:162-180, 2007

8. Farkas E, Obrenovitch TP, Institóris Á, Bari F: Effects of early aging and cerebral hypoperfusion on spreading depression in rats. Neurobiol Aging 32:1707-1715, 2011

9. Fluri F, Grünstein D, Cam E, Ungethuem U, Hatz F, Schäfer $J$, et al: Fullerenols and glucosamine fullerenes reduce infarct volume and cerebral inflammation after ischemic stroke in normotensive and hypertensive rats. Exp Neurol 265:142151,2015

10. Fukui M, Kono S, Sueishi K, Ikezaki K: Moyamoya disease. Neuropathology 20 Suppl:S61-S64, 2000

11. Hecht N, Schneider UC, Czabanka M, Vinci M, Hatzopoulos AK, Vajkoczy P, et al: Endothelial progenitor cells augment collateralization and hemodynamic rescue in a model of chronic cerebral ischemia. J Cereb Blood Flow Metab 34:1297-1305, 2014

12. Hiramatsu M, Hishikawa T, Tokunaga K, Kidoya H, Nishihiro S, Haruma J, et al: Combined gene therapy with vascular endothelial growth factor plus apelin in a chronic cerebral hypoperfusion model in rats. J Neurosurg 127:679-686, 2017

13. Houkin K, Ito M, Sugiyama T, Shichinohe H, Nakayama N, Kazumata K, et al: Review of past research and current concepts on the etiology of moyamoya disease. Neurol Med Chir (Tokyo) 52:267-277, 2012

14. Ishii R, Takeuchi S, Ibayashi K, Tanaka R: Intelligence in children with moyamoya disease: evaluation after surgical treatments with special reference to changes in cerebral blood flow. Stroke 15:873-877, 1984

15. Jung KH, Chu K, Lee ST, Park HK, Kim DH, Kim JH, et al: Circulating endothelial progenitor cells as a pathogenetic marker of moyamoya disease. J Cereb Blood Flow Metab 28:1795-1803, 2008

16. Kamada F, Aoki Y, Narisawa A, Abe Y, Komatsuzaki S, Kikuchi A, et al: A genome-wide association study identifies RNF213 as the first moyamoya disease gene. J Hum Genet 56:34-40, 2011

17. Kamata I, Terai Y, Ohmoto T: Attempt to establish an experimental animal model of moyamoya disease using immunoembolic material-histological changes of the arterial wall resulting from immunological reaction in cats. Acta Med Okayama 57:143-150, 2003

18. Kasai N, Fujiwara S, Kodama N, Yonemitsu T, Suzuki J: [The experimental study on causal genesis of moyamoya disease-correlation with immunological reaction and sympathetic nerve influence for vascular changes (author's transl).] No Shinkei Geka 10:251-261, 1982 (Jpn)

19. Kim JH, Jung JH, Phi JH, Kang HS, Kim JE, Chae JH, et al: Decreased level and defective function of circulating endothelial progenitor cells in children with moyamoya disease. J Neurosci Res 88:510-518, 2010

20. Kobayashi H, Matsuda Y, Hitomi T, Okuda H, Shioi H, Matsuda T, et al: Biochemical and functional characterization of RNF213 (mysterin) R4810K, a susceptibility mutation of moyamoya disease, in angiogenesis in vitro and in vivo. $\mathbf{J}$ Am Heart Assoc 4:e002146, 2015

21. Lee JY, Moon YJ, Lee HO, Park AK, Choi SA, Wang KC, et al: Deregulation of retinaldehyde dehydrogenase 2 leads to defective angiogenic function of endothelial colony-forming cells in pediatric moyamoya disease. Arterioscler Thromb Vasc Biol 35:1670-1677, 2015

22. Moubarik C, Guillet B, Youssef B, Codaccioni JL, Piercecchi MD, Sabatier F, et al: Transplanted late outgrowth endothelial progenitor cells as cell therapy product for stroke. Stem Cell Rev 7:208-220, 2011

23. Ni JW, Matsumoto K, Li HB, Murakami Y, Watanabe H: Neuronal damage and decrease of central acetylcholine level following permanent occlusion of bilateral common carotid arteries in rat. Brain Res 673:290-296, 1995

24. Pellegrini L, Bennis Y, Guillet B, Velly L, Garrigue P, Sabatier F, et al: Therapeutic benefit of a combined strategy using erythropoietin and endothelial progenitor cells after transient focal cerebral ischemia in rats. Neurol Res 35:937-947, 2013

25. Phi JH, Suzuki N, Moon YJ, Park AK, Wang KC, Lee JY, et al: Chemokine ligand 5 (CCL5) derived from endothelial colony-forming cells (ECFCs) mediates recruitment of smooth muscle progenitor cells (SPCs) toward critical vascular locations in moyamoya disease. PLoS One 12:e169714, 2017

26. Rafat N, Beck GCh, Peña-Tapia PG, Schmiedek P, Vajkoczy $P$ : Increased levels of circulating endothelial progenitor cells in patients with moyamoya disease. Stroke 40:432-438, 2009

27. Schrott LM, Franklin L, Serrano PA: Prenatal opiate exposure impairs radial arm maze performance and reduces levels of BDNF precursor following training. Brain Res 1198:132 140,2008

28. Sebastian V, Diallo A, Ling DS, Serrano PA: Robust training attenuates TBI-induced deficits in reference and working memory on the radial 8-arm maze. Front Behav Neurosci 7:38, 2013

29. Shantsila E, Blann AD, Lip GY: Circulating endothelial cells: from bench to clinical practice. J Thromb Haemost 6:865868,2008

30. Sherma AK, Bendok BR: Endothelial progenitor cells in moyamoya disease. Neurosurgery 64:N11, 2009

31. Sonobe T, Akiyama T, Du CK, Zhan DY, Shirai M: Contribution of calpain to myoglobin efflux from cardiomyocytes during ischaemia and after reperfusion in anaesthetized rats. Acta Physiol (Oxf) 210:823-831, 2014

32. Sutherland BA, Rabie T, Buchan AM: Laser Doppler flowmetry to measure changes in cerebral blood flow. Methods Mol Biol 1135:237-248, 2014

33. Trebst C, Heine S, Lienenklaus S, Lindner M, Baumgärtner W, Weiss S, et al: Lack of interferon-beta leads to accelerated remyelination in a toxic model of central nervous system demyelination. Acta Neuropathol 114:587-596, 2007

34. van Dijk A, Naaijkens BA, Jurgens WJ, Nalliah K, Sairras S, 
van der Pijl RJ, et al: Reduction of infarct size by intravenous injection of uncultured adipose derived stromal cells in a rat model is dependent on the time point of application. Stem Cell Res (Amst) 7:219-229, 2011

\section{Disclosures}

The authors report no conflict of interest concerning the materials or methods used in this study or the findings specified in this paper.

\section{Author Contributions}

Conception and design: Kim, Choi. Acquisition of data: Choi, Kwak, Moon, Jangra. Analysis and interpretation of data: Choi, Kwak, Park. Drafting the article: Choi, Chong. Critically revising the article: Kim, Choi, Phi, Lee, Park. Reviewed submitted version of manuscript: Choi. Study supervision: Kim.

\section{Supplemental Information}

Online-Only Content

Supplemental material is available with the online version of the article.

Supplemental Tables $S 1 \&$ S2. https://thejns.org/doi/suppl/ 10.3171/2018.8.PEDS1883.

\section{Correspondence}

Seung-Ki Kim: Seoul National University Children's Hospital, Seoul, Republic of Korea.nsthomas@snu.ac.kr. 